\title{
Anomalous preasymptotic colloid transport by hydrodynamic dispersion in microfluidic capillary flow
}

\author{
Einar Orn Fridjonsson, ${ }^{1, *}$ Joseph D. Seymour, ${ }^{1,2, \dagger}$ and Sarah L. Codd ${ }^{2,3}$ \\ ${ }^{1}$ Department of Chemical and Biological Engineering, Montana State University, Bozeman, Montana 59717-3920, USA \\ ${ }^{2}$ Center for Biofilm Engineering, Montana State University, Bozeman, Montana 59717-3980, USA \\ ${ }^{3}$ Department of Mechanical and Industrial Engineering, Montana State University, Bozeman, Montana 59717-3800, USA
}

(Received 2 April 2014; published 2 July 2014)

\begin{abstract}
The anomalous preasymptotic transport of colloids in a microfluidic capillary flow due to hydrodynamic dispersion is measured by noninvasive nuclear magnetic resonance (NMR). The data indicate a reduced scaling of mean squared displacement with time from the $\left\langle z(t)^{2}\right\rangle_{c} \sim t^{3}$ behavior for the interaction of a normal diffusion process with a simple shear flow. This nonequilibrium steady-state system is shown to be modeled by a continuous time random walk (CTRW) on a moving fluid. The full propagator of the motion is measured by NMR, providing verification of the assumption of Gaussian jump length distributions in the CTRW model. The connection of the data to microrheology measurements by NMR, in which every particle in a suspension contributes information, is established.
\end{abstract}

DOI: 10.1103/PhysRevE.90.010301

PACS number(s): 82.70.-y, 83.80.Hj, 82.56.Lz, 05.40.Fb

The dynamics of colloidal particles suspended in liquids in small channels impact mammalian physiology [1], design of microfluidic sensors [2], and transport in geological systems [3]. Colloids are also a model system for understanding the molecular behavior of dense fluids, glass phase transitions, and nonequilibrium statistical mechanics [4]. The displacement time dependent dynamics of colloids in small channels control physiological transport in the microvasculature [1] and the ability to probe a particle with a physical or chemical assay in microfluidic sensors [2]. Hydrodynamic dispersion [5], the interaction of advective and diffusive displacements, determines the transit time of a channel, and is characterized by the axial mean squared displacement (MSD). Despite extensive research on colloid particle dynamics and well established theory for the time dependent hydrodynamic dispersion of single particles, limited data or theory exist for the time dependent dispersive transport of concentrated colloids in microchannels. Here we present unique data directly measuring the displacement time dependent MSD of concentrated colloids in a microcapillary using noninvasive nuclear magnetic resonance (NMR). The data indicate a shear dependent structural rearrangement [6,7]. Particles first break out of a cage of neighboring particles [8] and then undergo dynamics [7] generating anomalous transport with axial MSD $\left\langle z(t)^{2}\right\rangle_{c} \sim t^{\alpha}$ where $\alpha \sim 2.5$. These dynamics are in contrast to normal diffusion driven preasymptotic hydrodynamic dispersion where $\alpha=3$. Mode coupling theory for infinite spatial extent sheared glasses also predicts an $\alpha=3$ MSD scaling for the flow direction [9]. A continuous time random walk (CTRW) on moving fluids theory [10] is demonstrated to model concentrated colloid hydrodynamic dispersion in microfluidic flow.

\footnotetext{
*Present address: School of Mechanical and Chemical Engineering, University of Western Australia, Crawley, WA, Australia.

${ }^{\dagger}$ Author to whom correspondence should be addressed: jseymour@coe.montana.edu
}

The transport of a colloid particle in capillary flow is determined by the classic Taylor or hydrodynamic dispersion [5], in which particle diffusion randomly samples the deterministic velocity field [11]. The long time asymptotic behavior of hydrodynamic dispersion is a normal diffusion process in which the particle MSD behaves as $\left\langle z(t)^{2}\right\rangle_{c}=2 D^{*} t$ for times $t \gg \tau_{D}=R^{2} / D_{o}$. The hydrodynamic dispersion coefficient for capillary flow $D^{*}=\left\langle v_{z}\right\rangle R / 48 D_{o}$ depends on the average velocity $\left\langle v_{z}\right\rangle$, the capillary radius $R$, and the diffusion constant $D_{o}$ by which the tracer particle samples the velocity field [5]. In microfluidic applications where the capillary radius $R$ is small $(\sim 100 \mu \mathrm{m})$, the time for a liquid molecule to completely sample the velocity field by random diffusion $D_{o} \sim 10^{-9}$ $\mathrm{m}^{2} / \mathrm{s}$ is $\tau_{D} \sim R^{2} / D_{o} \sim 10^{1} \mathrm{~s}$. In contrast, the diffusion time scale for transport across the capillary for a radius $a \sim 1 \mu \mathrm{m}$ colloid of slower diffusivity given by the Stokes-EinsteinSutherland diffusion coefficient $\left(D_{o}=D_{\mathrm{SES}}=k T / 6 \pi \mu a \sim\right.$ $10^{-13} \mathrm{~m}^{2} / \mathrm{s}$ ) is $\tau_{D} \sim 10^{5} \mathrm{~s}$. This long time to sample the velocity field by diffusion indicates preasymptotic dynamics controls colloid transport in microfluidic systems. The time dependent axial motion in the direction of flow is characterized by the variance, or MSD, of the time dependent particle position $z(t)$. For a simple two-dimensional (2D) linear shear field, the axial velocity $v_{z}=G x$ varies linearly in the $x$ position with constant shear rate $G=d v_{z} / d x$ and the MSDs are $\left\langle x(t)^{2}\right\rangle=2 D_{o} t,\langle x(t) z(t)\rangle=2 D_{o}\left(\frac{1}{2} G t^{2}\right)$, and $\left\langle z(t)^{2}\right\rangle_{c}=$ $\left\langle z(t)^{2}\right\rangle-\langle z(t)\rangle^{2}=2 D_{o}\left(t+\frac{1}{3} G^{2} t^{3}\right)$. This result has been derived using a multitude of analytical methods based on the advection diffusion equation [11-15], the projection operator method [16], and the generalized Langevin equation $[11,15,17,18]$. Direct numerical simulations have recently reproduced this time and shear rate dependent behavior [19]. It has also recently been observed experimentally in a cone and plate rotational flow by confocal microscopy [20]. Flow in a pipe with uniform initial distribution of particles results in identical scaling for the axial MSD [15,17].

A model of particle hydrodynamic dispersion in a shear flow has been formulated in terms of a continuous time random walk (CTRW) on a moving fluid [10]. CTRW models are 


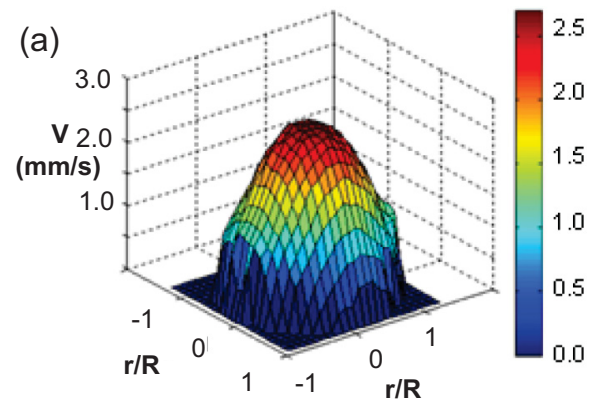

(c)

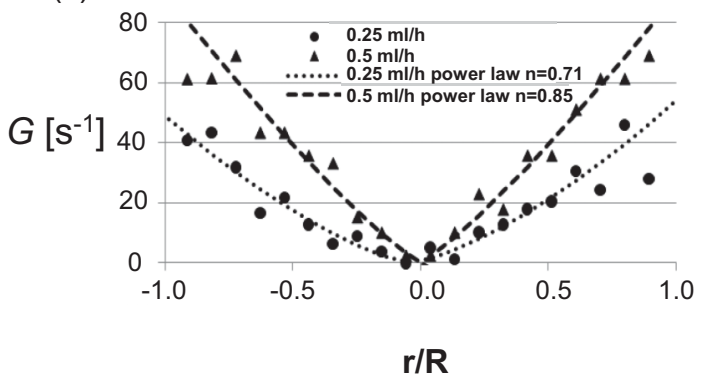

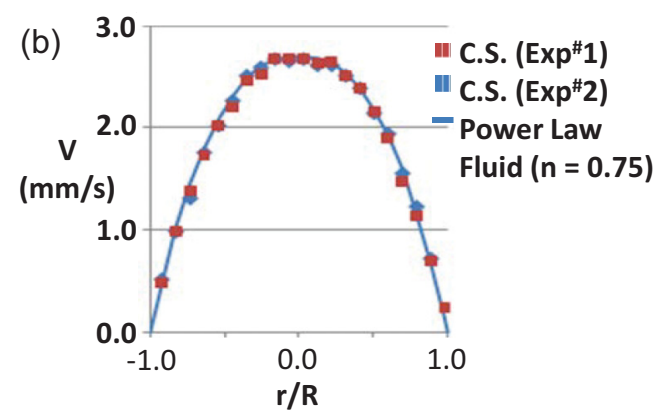

(d)

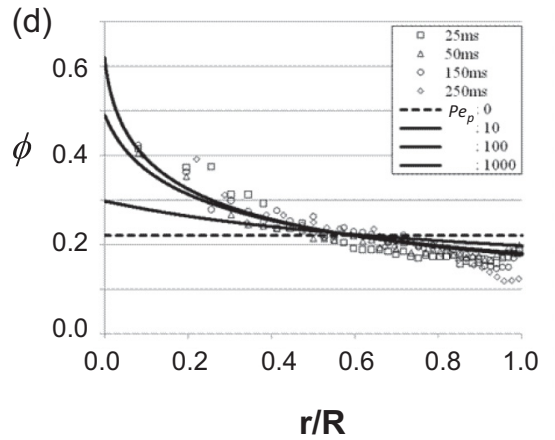

FIG. 1. (Color online) Magnetic resonance images of the spatial velocity dependence characterize the macroscale rheology of the fluid and allow calculation of the nonequilibrium steady-state momentum and mass flux driving forces. The MRI measured spatial phase averaged $\left\langle v_{z}(r)\right\rangle=\phi\left\langle v_{p}(r)\right\rangle+(1-\phi)\left\langle v_{f}(r)\right\rangle$ velocity distribution at a volumetric flow rate of $Q=0.25 \mathrm{ml} / \mathrm{h}$ with an in plane spatial image resolution of $11.7 \mu \mathrm{m} /$ pixel is shown in (a). The velocity profile along a diameter in (b) allows determination of the power-law fluid rheology of the suspension. The velocity profile data derivative with respect to radial position provides the spatial shear rate distribution, shown in (c) for $Q=0.25$ and $0.5 \mathrm{ml} / \mathrm{h}$ and compared to the power-law rheology fluid form. The particle concentration profile in (d) is measured (open symbols) by mapping the spatial distribution of velocity to the independently measured probability distribution of colloid velocity. The solid lines [29] are a normal pressure based model for various particle Peclet numbers.

based on a probability $\Psi(Z, t)=\psi_{o}(Z) \phi_{o}(t)$ that a random walker makes a jump of length $Z$ after waiting time $t$ $[10,21]$. The CTRW model on a moving fluid uses a Gaussian distribution of jump lengths $\psi_{o}(Z)=\exp \left(-Z^{2} / 2 \sigma^{2}\right)$ with variance $\sigma^{2}$ and power-law Levy distributed wait time $\phi_{o}(t)=$ $\tau t^{-1-\beta}$. The power-law wait time distribution is due to the correlated dynamics of the particles. The CTRW on a moving fluid generates a time fractional advection diffusion equation governing the axial flow direction transport of the colloid particle [10,21]. The axial MSD for this CTRW model in a shear flow is $\left\langle z(t)^{2}\right\rangle_{c}=2 D\left(\frac{t^{\beta}}{\Gamma(1+\beta)}+\frac{2}{\Gamma(1+3 \beta)} G^{2} t^{3 \beta}\right)$, which returns the result for normal diffusion for $\beta=1$, corresponding to a Poisson wait time distribution [10]. The physical origin of the Levy wait time model in a colloid suspension is long range correlated dynamics induced by dynamical heterogeneity and structural rearrangements of the particles [6-8,22].

Pulsed gradient spin echo (PGSE) NMR allows direct measurement of the separate affine and random particle motions, providing unique data on hydrodynamic dispersion $[23,24]$. In particular, the ability to velocity compensate the NMR signal by application of specific magnetic field gradient wave forms removes the effect of affine dynamics. This allows direct measurement of random motions [24]. Core shell polymethylmethacrylate (PMMA) particles of radius $a=1.25 \pm 0.46 \mu \mathrm{m}$ filled with hexadecane oil and suspended in a water and polyvinyl alcohol (PVA) solution [25] permit separate measurements of the colloid particle and suspending fluid dynamics. The dynamics of the suspended particles and suspending fluid phases are measured simultaneously by resonant frequency resolution of the oil and water peaks of each phase [26]. Figure 1(a) shows magnetic resonance image (MRI) data of the spatial velocity distribution measured by velocity sensitive PGSE MRI for a colloid suspension of solids volume fraction $\phi=0.22$, flowing at $Q=0.25 \mathrm{ml} / \mathrm{h}$ in a capillary of radius $R=126 \mu \mathrm{m}$. In this data spectral resolution of the oil (particle) and water (suspending fluid) is not obtained due to the imaging gradient. Thus, the spatial phase averaged velocity $\left\langle v_{z}(r)\right\rangle=\phi\left\langle v_{p}(r)\right\rangle+(1-\phi)\left\langle v_{f}(r)\right\rangle$ is measured in the suspension at each point in the image. Measurements of pure water exhibit the classic parabolic spatial velocity distribution $v_{z}(r)=v_{z, \max }\left[1-(r / R)^{2}\right]$ for low tube Reynolds number, $\operatorname{Re}_{t}=\rho v_{z, \max } R / \mu \sim 0.35$, which is the laminar pressure driven flow of a constant viscosity Newtonian fluid in a capillary. In contrast, the suspension velocity profile in Fig. 1(a) is blunted $v_{z}(r)=v_{z, \max }\left[1-(r / R)^{(1 / n)+1}\right]$ due to power-law shear rate dependent viscosity $\eta(G) \sim(G)^{(n-1)}$ with power-law index $n$, where $n=1$ for a Newtonian fluid. The average shear rate in the system is $G=-4 v_{z, \max } / 3 R$. The macroscale rheology of the suspension is measured directly by the velocity imaging by determination of the spatial velocity distribution Fig. 1(b) and shear rate distribution Fig. 1(c). The measured power-law index is calculated to be $n=0.73 \pm$ 0.02 at $Q=0.25 \mathrm{ml} / \mathrm{h}$. The data provides characterization of the macroscale rheology by rheo-NMR [27]. The power-law 
fluid blunting of the velocity distribution is known to have little impact on the preasymptotic hydrodynamic dispersion behavior discussed below [28].

The flow is well developed before the NMR detection region. An entrance length $L_{e}>2000 R$ assures a steady-state particle distribution due to shear induced diffusion, which generates a higher particle concentration near the capillary center [29]. The volume fraction was chosen so that many particle hydrodynamic interactions would be present but complete jamming in the tube center would not occur. The concentration gradient $d \phi / d r$ measured using the NMR data [30] varies from $\phi=0.45$ near the capillary center to $\phi=0.15$ near the wall. The impact of the radial particle concentration distribution on asymptotic hydrodynamic dispersion, for times longer than it takes to move a correlation length $L_{c}=3 R^{3} / 8 a^{2}$, recently has been shown to generate non-Gaussian dispersion [31]. In our system, the maximum length over which the particles displace during a measurement is the sensitive region of the NMR coil, $1 \times 10^{-2} \mathrm{~m}$. This is far below the correlation length of $L_{c} \sim 0.48 \mathrm{~m}$. Hence the shear induced spatial particle distribution has limited impact on our hydrodynamic dispersion data.

To directly measure the axial colloid $\operatorname{MSD}\left\langle z(t)^{2}\right\rangle_{c}$, velocity compensated PGSE NMR data [24] are obtained using large magnetic field gradients of up to $11.3 \mathrm{~T} / \mathrm{m}$. PGSE NMR directly measures the diffusion coefficient of all nuclei with gyromagnetic ratio $\gamma$ in the NMR radio frequency coil as a function of experimentally controlled displacement time $t=\Delta$ through attenuation of the NMR voltage signal $E(\Delta)=\exp \left[-\gamma^{2} g^{2} \delta^{2} D^{*}(\Delta-\delta / 3)\right]$. The magnetic field gradient pulse of duration $\delta$ is incremented in amplitude $g$ and the signal attenuation as a function of $g$ fit to determine $D^{*}$ [24]. It is informative to analyze the effective diffusion, or hydrodynamic dispersion, coefficient $D^{*}(t)=\left\langle z(t)^{2}\right\rangle_{c} / 2 t$ as well as the MSD. In the absence of flow at short times $t<a^{2} / D_{\text {oil }} \sim 2.2 \mathrm{~ms}$, the oil in the core shell particle has not diffused a long enough distance to sample the confining core shell. The effective diffusion is then the diffusion coefficient of the free hexadecane oil $D^{*}(t)=D_{\text {oil }}=4.6 \times 10^{-10} \mathrm{~m}^{2} / \mathrm{s}$. At longer times $t>a^{2} / D_{\text {oil }}$, the effective time dependent diffusion coefficient decreases as displacement observation time is increased, due to restricted diffusion in the spherical pore formed by the core shell. This effect is well known in measurements of porous media [24,25]. In a system of spherical pores with a near Gaussian radius distribution, such as our particles, theory predicts $D^{*}(t) \sim a^{2} / 5 t$. The fit to this is shown as a black line for the no flow $0.0 \mathrm{ml} / \mathrm{h}$ data in Fig. 2(a) and provides measurement of the core shell particle inner radius.

The impact of shear flow on the axial colloid particle hydrodynamic dispersion is investigated using three flow rates $Q=0.125,0.250$, and $0.500 \mathrm{ml} / \mathrm{h}$ corresponding to shear rates $G=13.7,27.1$, and $59.2 \mathrm{~s}^{-1}$. The flow rates correspond to low particle Reynolds numbers $\operatorname{Re}=\rho a^{2} G / \mu=2.3 \times$ $10^{-5}, 4.6 \times 10^{-5}$, and $9.3 \times 10^{-5}$, indicating particle inertia is negligible. The particle Peclet numbers $\mathrm{Pe}=a^{2} G / D_{\mathrm{SES}}=80$, 160,321 signify that the advective transport rate dominates the diffusive transport rate. In the presence of shear flow, the effective diffusion coefficient [Fig. 2(a)] decreases until a displacement time equivalent to a total strain $\gamma=G t \sim 1$ [32]. (a)

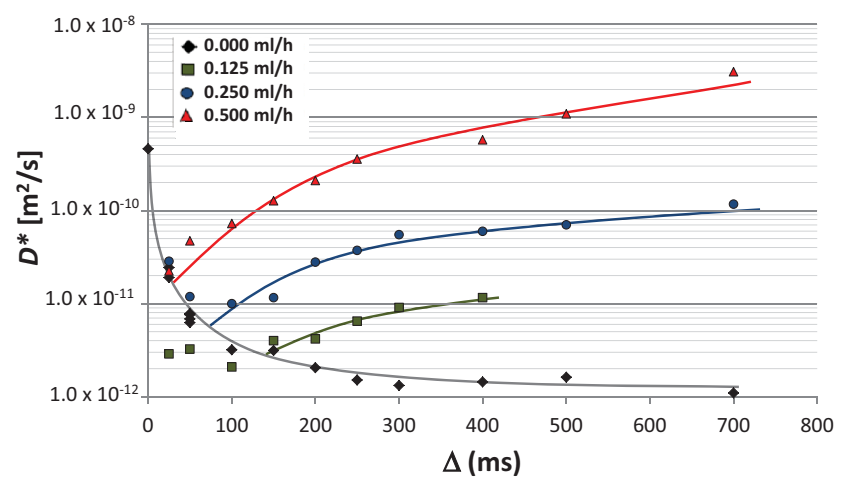

(b)

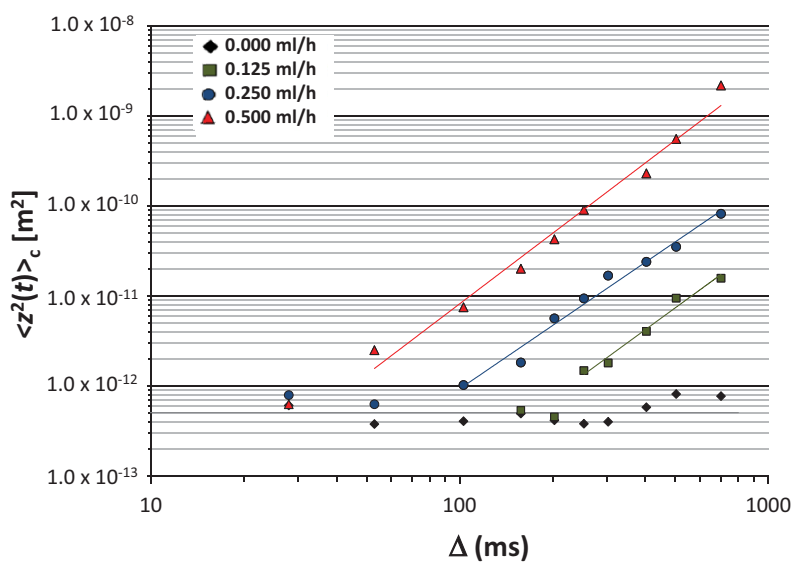

FIG. 2. (Color online) (a) The MR measured time dependent axial diffusion coefficient and (b) mean square displacement (MSD) as a function of experimental displacement observation time $(\Delta)$ and volumetric flow rates $Q=0.0,0.125,0.250$, and $0.500 \mathrm{ml} / \mathrm{h}$ corresponding to average shear rates $G=0.0,13.7,27.1$, and $59.2 \mathrm{~s}^{-1}$. The MSD data is fit using a power-law scaling $\left\langle z^{2}(t)\right\rangle_{c} \sim \Delta^{\alpha}$ to obtain $\alpha \sim 2.5 \pm 0.1$ for all three flowing conditions. The MSD of the hexadecane molecules inside the spheres for the no flow condition is obtained using $\left\langle z^{2}(t)\right\rangle_{c} \sim \frac{2}{5} a^{2}$, providing the mean internal size of the spheres from the theory of MR diffusion measurements in porous media [24].

The effective diffusion coefficient for longer displacement times $t>1 / G$ increases with time due to the axial random particle motions. At displacement times $t<1 / G$ the impact on the NMR signal of the random particle motions is smaller than molecular diffusion of the oil within the particles, while for displacement times $t>1 / G$ the particles undergo larger random axial displacements due to hydrodynamic dispersion. This indicates the colloid particles are caged [8] by neighbor particles at times $t<1 / G$ and break out of the cage at time $t \sim 1 / G$, in agreement with simulations [6].

To analyze the increase in random dynamics at times $t>1 / G$, the MSD displacement as a function of displacement time is plotted in Fig. 2(b) and is fit to $\left\langle z(t)^{2}\right\rangle_{c} \sim t^{\alpha}$ with $\alpha \sim 2.5 \pm 0.1$. This indicates anomalous preasymptotic hydrodynamic dispersion. Note that some authors [14,20] have termed the $\left\langle z(t)^{2}\right\rangle_{c} \sim t^{3}$ behavior of preasymptotic hydrodynamic dispersion as anomalous. However, since this behavior 

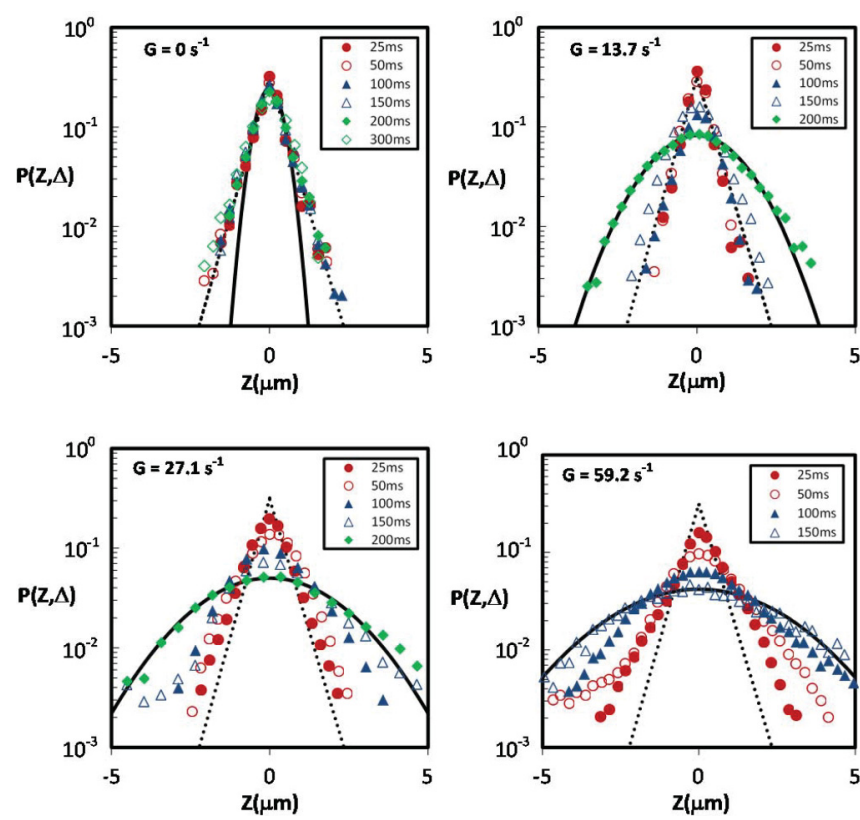

FIG. 3. (Color online) The propagator of the axial colloid motion, i.e., the probability $P(Z, \Delta)$ that axial colloid displacements $Z$ occur in time $\Delta$, measured as a function of displacement observation time $\Delta$ and shear rate. Under no flow conditions, $G=0 \mathrm{~s}^{-1}$ in the $\phi=22 \%$ volume fraction suspension, the restricted motion of the internal hexadecane is evident by the non-Gaussian propagators of fixed width. The solid line is a Gaussian fit and the dashed line is an exponential fit. The sheared suspensions display similar non-Gaussian restricted motion for displacement time $\Delta<1 / G$ due to caging of the particles. The dashed lines on the plots for the sheared suspensions are the exponential fit to the no flow propagators. The solid lines are Gaussian fits to the longest displacement time data for each shear rate $G$. The propagators transition to Gaussian distributions at displacement times $\Delta>1 / G$, confirming the CTRW model of jump lengths being Gaussian.

is due to a normal diffusion interacting with the deterministic velocity shear field at short times, we refer to it as normal preasymptotic hydrodynamic dispersion. The $\left\langle z(t)^{2}\right\rangle_{c} \sim t^{\alpha}$, $\alpha \neq 3$ behavior is termed anomalous, in keeping with the approach outlined by Metzler and Klafter [21]. In the CTRW model the anomalous transport decreases the time scaling relative to the normal preasymptotic dispersion, i.e., $\alpha<3$. The correlated dynamics modeled by the power-law wait time distribution $\phi_{o}(t)=\tau t^{-1-\beta}$ decreases the axial spreading in time. The NMR data indicate this reduced axial spreading with time scales as $\alpha \sim 2.5 \pm 0.1$. The wait time distribution is $\phi_{o}(t) \sim t^{-1.8}$ [33], which characterizes the particle dynamic correlations caused by microstructure rearrangements, i.e., dynamical heterogeneities [6]. An interesting aspect of the MSD data is that they represent a microrheology [33,34] measurement by NMR, $\mu$-rheo-NMR. All particles in the suspension contribute to the measured MSD, in contrast to most microrheological measurements which utilize a single tracer particle. Recently confocal microscopy has been used to measure MSD in the vorticity direction of transient flows to correlate microrheology to stress overshoot in concentrated colloidal suspensions [35]. In contrast, our data characterize transient dynamics in a steady-state flow and incorporation of these axial transport dynamics in a microrheological model has the potential to provide different insight into the microrheology of colloid suspensions.

The nanometer resolution of the displacement time scale dependent dynamics and the structural rearrangement are elegantly demonstrated by the propagator of the motion [24] $P(Z, t)$, measured by the velocity compensated PGSE NMR measurement. The propagators in Fig. 3 are centered at zero displacement $Z(t)=z(t)-z(0)$ since the affine displacement is refocused. The no flow propagators are non-Gaussian due to the restricted motion of the oil within the particles and are fit to an exponential distribution. The no flow propagator provides a measure of the particle size from the half-height width equal to $a \sim 1.25 \mu \mathrm{m}[24,36]$. In the presence of shear, the displacement probabilities indicate limited change in width until $t \sim 1 / G$. They then transition from the non-Gaussian restricted motion distribution of displacements to near Gaussian as the particles break out of the restricting cage of neighboring particles. The propagators provide direct measurement of the Gaussian jump length distribution, validating the CTRW model assumption of independence of the wait time and jump length distribution.

PGSE NMR measurements of preasymptotic hydrodynamic dispersion for a concentrated colloidal suspension in microfluidic shear flow exhibit anomalous displacement dynamics. The axial spreading is slower than for normal preasymptotic hydrodynamic dispersion. The colloid axial MSD is shown to be modeled by CTRW theory [10]. The measured dynamics indicate structural rearrangements occur at times $t \sim 1 / G$, in agreement with simulations of dynamical heterogeneities in sheared systems [6]. The data demonstrate the ability of NMR to noninvasively quantify macroscale and microscale colloid dynamics in three-dimensional (3D) shear flows. The NMR measurements provide different data for microrheological analysis and indicate that colloid transport in physiological and geological applications, as well as design of microfluidic devices, can benefit from the application of CTRW models.

The authors acknowledge Sir Paul T. Callaghan for guidance. This research was supported in part by the NSF CAREER Award to J.D.S. (No. CTS-0348076) and the NSF CAREER Award to S.L.C. (No. CBET-0642328). The M. J. Murdock Charitable Trust and NSF MRI program provided equipment funding.
[1] G. R. Cokelet and H. L. Goldsmith, Circ. Res. 68, 1 (1991).

[2] T. M. Squires and S. R. Quake, Rev. Mod. Phys. 77, 977 (2005).

[3] A. B. Kersting et al., Nature (London) 397, 56 (1999).
[4] P. J. Lu and D. A. Weitz, Annu. Rev. Condens. Matter Phys. 4, 217 (2013).

[5] G. I. Taylor, Proc. R. Soc. London, Ser. A 219, 186 (1953). 
[6] K. Martens, L. Bocquet, and J.-L. Barrat, Phys. Rev. Lett. 106, 156001 (2011).

[7] A. M. Leshansky, J. F. Morris, and J. F. Brady, J. Fluid Mech. 597, 305 (2008).

[8] P. M. Reis, R. A. Ingale, and M. D. Shattuck, Phys. Rev. Lett. 98, 188301 (2007).

[9] M. Kruger, F. Weysser, and M. Fuchs, Eur. Phys. J. E 34, 88 (2011).

[10] A. Compte, Phys. Rev. E 55, 6821 (1997).

[11] R. T. Foister and T. G. M. Van De Ven, J. Fluid Mech. 96, 105 (1980).

[12] D. E. Elrick, Aust. J. Phys. 15, 283 (1962).

[13] G. K. Batchelor, J. Fluid Mech. 95, 369 (1979).

[14] M. Latini and A. J. Bernoff, J. Fluid Mech. 441, 399 (2001).

[15] R. Camassa, Z. Lin, and R. M. McLaughlin, Commun. Math. Sci. 8, 601 (2010).

[16] M. San Miguel and J. M. Sancho, Physica A 99, 357 (1979).

[17] C. van den Broeck, Physica A 112, 343 (1982).

[18] K. Miyazaki and D. Bedeaux, Physica A 217, 53 (1995).

[19] T. Iwashita and R. Yamamoto, Phys. Rev. E 79, 031401 (2009).

[20] H. Orihara and Y. Takikawa, Phys. Rev. E 84, 061120 (2011).

[21] R. Metzler and J. Klafter, Phys. Rep. 339, 1 (2000).

[22] J. Goyon, A. Colin, G. Ovarlez, A. Ajdari, and L. Bocquet, Nature (London) 454, 84 (2008).
[23] S. L. Codd, B. Manz, J. D. Seymour, and P. T. Callaghan, Phys. Rev. E 60, R3491 (1999).

[24] P. T. Callaghan, Translational Dynamics and Magnetic Resonance Principles of Pulsed Gradient Spin Echo NMR (Oxford University Press, Oxford, UK, 2011).

[25] H. Wassenius, M. Nyden, and B. Vincent, J. Colloid Interface Sci. 264, 538 (2003).

[26] J. R. Brown et al., Phys. Rev. Lett. 99, 240602 (2007).

[27] P. T. Callaghan, Curr. Opin. Colloid Interface Sci. 11, 13 (2006).

[28] G. S. Booras and W. B. Krantz, Ind. Eng. Chem. Fundam. 15, 249 (1976).

[29] D. Semwogerere, J. F. Morris, and E. R. Weeks, J. Fluid Mech. 581, 437 (2007).

[30] J. R. Brown, E. O. Fridjonsson, J. D. Seymour, and S. L. Codd, Phys. Fluids 21, 093301 (2009).

[31] I. M. Griffiths and H. A. Stone, Europhys. Lett. 97, 58005 (2012).

[32] D. J. Pine, J. P. Gollub, J. F. Brady, and A. M. Leshansky, Nature (London) 438, 997 (2005).

[33] T. G. Mason and D. A. Weitz, Phys. Rev. Lett. 74, 1250 (1995).

[34] T. M. Squires and T. G. Mason, Annu. Rev. Fluid Mech. 42, 413 (2010).

[35] M. Laurati et al., J. Phys.: Condens. Matter 24, 464104 (2012).

[36] D. G. Cory and A. N. Garroway, Magn. Reson. Med. 14, 435 (1990). 